\title{
A BEM method for electromagnetics in complex 3D geometries
}

\author{
P. L'Eplattenier \& I. Çaldichoury \\ Livermore Software Technology Corporation, USA
}

\begin{abstract}
An electromagnetism module is under development in the commercial software LS-DYNA in order to perform coupled mechanical/thermal/electromagnetic simulations. The main part of the module is a so-called "eddy current" solver. This module allows us to introduce source electrical currents into solid conductors, and to compute the associated magnetic fields, electric fields, induced currents, Lorentz forces, Joule heating and so forth. The Maxwell equations are solved using a Finite Element Method (FEM) for the solid conductors coupled with a Boundary Element Method (BEM) for the surrounding air (or insulators). Both the FEM and the BEM are based on discrete differential forms (Nedelec-like elements). The use of the BEM for the air allows to handle complex 3D geometries with multiply connected conductors, very small air gaps, and motion of the conductors, due, in particular, to the electromagnetic forces. The variable of the BEM is a socalled "surface current" allowing the connection of the model to external current sources by simple dirichlet constraints, as well as direct computations of the self and mutual inductances of the system. In order to handle the large and dense BEM matrices, a domain decomposition is performed and low rank approximations are done on the off-diagonal blocks of the resulting block matrices. The diagonal blocks are used as an efficient preconditioner when solving the BEM part of the system. The singularities arising in the computation of the self and neighbor matrix elements of the Galerkin BEM are taken into account using a method based on Duffy transforms. The BEM method will be presented as well as benchmarks and real life application examples.

Keywords: eddy current, finite element method, boundary element method, Nedelec elements, contact, coupled mechanical/thermal/electromagnetic simulations.
\end{abstract}




\section{Introduction}

LS-DYNA is a highly advanced general-purpose nonlinear finite element program that is capable of simulating complex real world problems. It is suitable to investigate phenomena that involve large deformations, sophisticated material models and complex contact conditions. LS-DYNA allows running an analysis explicitly or implicitly and combining different disciplines such as coupled thermal analysis, fluid dynamics, fluid-structure interaction, SPH (smooth Particle Hydrodynamics), EFG (Element Free Galerkin) [1]. An electromagnetism (EM) module is under development in LS-DYNA in order to perform coupled mechanical/thermal/electromagnetic simulations [2]. This module allows us to introduce some source electrical currents into solid conductors, and to compute the associated magnetic field, electric field, induced currents, Joule heating and electromagnetic (Lorentz) force. These fields are computed by solving the Maxwell equations in the eddy current approximation. The eddy current solver is the main part of the EM module, although an induced heating and resistive heating solvers have also been added. Since the EM module is part of a commercial software, it is used intensively in all sorts of geometries and with all kinds of meshes, some of them being of rather poor quality. A lot of effort was thus spent making the numerical method robust enough. On the bright side, we get extensive benchmarks and a lot of feedback on the method. In order to best handle the motion of the conductors without the need to remesh the air surrounding them, the electromagnetic fields are solved using a Finite Element Method (FEM) coupled with a Boundary Element Method (BEM). Since the FEM is based on differential forms, the BEM also is based on this same representation, in order to have a correspondence between the FEM and BEM basis functions at the surface of the FEM mesh, which corresponds to the BEM mesh.

In this paper, we will first present the physical problem and give a brief overview of the FEM used, and we will then focus in more details on the BEM, and in particular on the FEM/BEM coupling, the treatment of the singular integrals, the BEM matrices storage and solve and the contact. We will then show the different capabilities of the BEM on industrial and academic examples.

\section{Presentation of the problem}

\subsection{Physical problem}

The following is a summary of what is presented in more details in [2]. Let $\Omega$ be a set of multiply connected conducting regions. The surrounding insulator exterior regions will be called $\Omega_{e}$. The boundary between $\Omega$ and $\Omega_{e}$ is called $\Gamma$. In the following, we will denote $\vec{n}$ as the outward normal to surfaces $\Gamma$. The electrical conductivity, permeability and permittivity are called $\sigma, \mu$ and $\epsilon$ respectively. In $\Omega_{e}$, we have $\sigma=0$ and $\mu=\mu_{0}$. For all our applications, we can use the low frequency or "eddy current" approximation, valid for good enough conductors with 
low frequency varying fields such that the condition

$$
\epsilon \frac{\partial \vec{E}}{\partial t} \ll \sigma \vec{E}
$$

is satisfied, where $\vec{E}$ is the electric field. This approximation implies a divergence free current density and no free charge accumulation. Also, in all our cases, the permittivity is equal to the vacuum one: $\epsilon=\epsilon_{0}$.

In the eddy current approximation, the Maxwell equations read:

$$
\begin{array}{rc}
\vec{\nabla} \times \vec{E}=-\frac{\partial \vec{B}}{\partial t} & \text { (Faraday) } \\
\vec{\nabla} \times \frac{\vec{B}}{\mu}=\vec{j} & \text { (Ampere) } \\
\nabla \bullet \vec{B}=0 & \text { (Gauss-magnetism) } \\
\nabla \bullet \epsilon \vec{E}=0 & \text { (Gauss-electricity) } \\
\nabla \bullet \vec{j}=0 & \text { (Gauss) } \\
\vec{j}=\sigma \vec{E} & \text { (Ohm) } \\
\vec{B}=\mu \vec{H} & \text { (Constitutive eq.) }
\end{array}
$$

where $\vec{B}$ is the magnetic flux density and $\vec{j}$ the current density. The divergence condition (4) allows to introduce the magnetic vector potential $\vec{A}$ such that

$$
\vec{B}=\vec{\nabla} \times \vec{A}
$$

Using equation (2), we can then write the electric field as:

$$
\vec{E}=-\vec{\nabla} \phi-\frac{\partial \vec{A}}{\partial t}
$$

where $\phi$ is the so-called electric scalar potential [3]. When using the Gauge condition:

$$
\nabla \bullet \sigma \vec{A}=0
$$

equations (3), (6), (7), (9), (10) and (11) imply:

$$
\begin{aligned}
\nabla \bullet \sigma \vec{\nabla} \phi & =0 \\
\sigma \frac{\partial \vec{A}}{\partial t}+\vec{\nabla} \times \frac{1}{\mu} \vec{\nabla} \times \vec{A} & =-\sigma \vec{\nabla} \phi
\end{aligned}
$$

These two equations, along with suitable boundary conditions give the time evolution of $\vec{A}$ and $\phi$, from which we get the electromagnetic fields by (10), (9) and (7). 


\subsection{General numerical method: FEM coupled with BEM}

(12) is a Laplace equation and (13) a diffusion equation, hence the other name "diffusion-induction", sometimes used for the eddy current model (we shall see later about the "induction" part). Since these equations mostly deal with exterior derivatives of vector fields, we decided to use a finite element method based on differential forms, often referred as "edge elements" or "Nedelec elements" [4]. More precisely, we are using the FEMSTER library [5] which provides a discrete numerical implementation of the exterior derivatives, gradient, curl and divergence, and the corresponding elemental matrices on hexahedral, prismatic and tetrahedral elements [6]. In this representation, each electromagnetic field is represented by a certain type of form, depending on how it appears in the equations, forming a so-called "Tonti" diagram [7,8]. Figure 1 represents the position of the different fields of the eddy current model in a Tonti diagram, and Table 1 gives some details about the different forms.

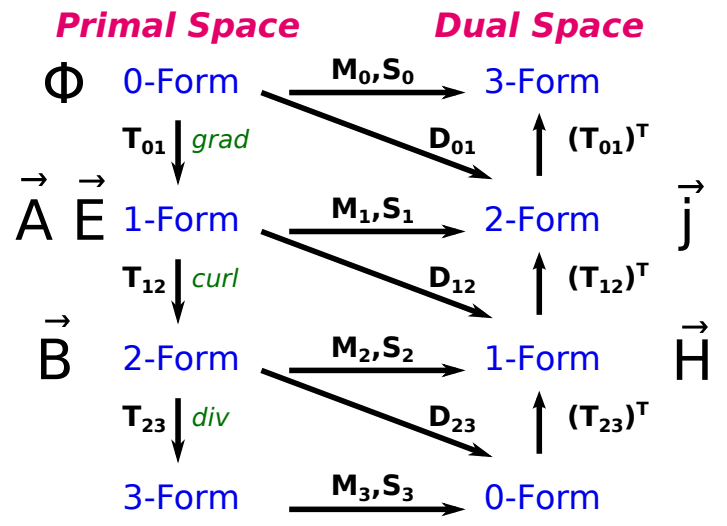

Figure 1: Tonti diagram for the eddy current problem showing the different forms, matrices and position of the EM fields.

Table 1: Definition of the $l$-forms, along with their Degrees Of Freedom (DOFs).

\begin{tabular}{cccc}
\hline Form type & Associated with & DOFs & Basis \\
\hline 0-form & Nodes & Nodal value & $W_{0}$ \\
1-form & Edges & Line integral & $\vec{W}_{1}$ \\
2-form & Faces & Flux & $\vec{W}_{2}$ \\
3-form & Cells & Volume integral & $W_{3}$ \\
\hline
\end{tabular}


The basis functions define spaces with an exact representation of the De-Rham sequence [6]. They also exactly satisfy numerical relations such as $\operatorname{curl}(\mathrm{grad})=0$ or $\operatorname{div}(\operatorname{curl})=0$. The basis functions associated respectively with the $0,1,2$, and 3- forms will be noted $W_{0}, \vec{W}_{1}, \vec{W}_{2}$, and $W_{3}$. Equation (12) is integrated against the $W^{0}$ forms and equation (13) against the $\vec{W}^{1}$ forms giving, after integrating by part, the following weak formulations [9]:

$$
\begin{aligned}
& \int_{\Omega} \sigma \vec{\nabla} \phi \cdot \vec{\nabla} W^{0} d \Omega=0 \\
& \begin{aligned}
\int_{\Omega} \sigma \frac{\partial \vec{A}}{\partial t} \cdot \vec{W}^{1} d \Omega & +\int_{\Omega} \frac{1}{\mu}(\vec{\nabla} \times \vec{A}) \cdot\left(\vec{\nabla} \times \vec{W}^{1}\right) d \Omega= \\
& \quad \int_{\Omega} \sigma \vec{\nabla} \Phi \cdot \vec{W}^{1} d \Omega+\int_{\Gamma} \frac{1}{\mu}[\vec{n} \times(\vec{\nabla} \times \vec{A})] \cdot \vec{W}^{1} d \Gamma
\end{aligned}
\end{aligned}
$$

which, after projecting $\phi$ on the $W_{0}$ forms and $\vec{A}$ on the $\vec{W}_{1}$ forms, give the FEM linear systems, which we write, with the same notations as in Figure 1, as:

$$
\begin{aligned}
\mathbf{S}^{0}(\sigma) \phi & =0 \\
\mathbf{M}^{1}(\sigma) \frac{d a}{d t}+\mathbf{S}^{1}\left(\frac{1}{\mu}\right) a & =-\mathbf{D}^{01}(\sigma) \phi+\mathbf{S} a
\end{aligned}
$$

\section{Introduction of the BEM}

\subsection{Presentation of the method}

The goal of the BEM is to compute the last surface term of (15), or (17), i.e. $\mathbf{S} a$. This term represents the interactions between the different conductors through the EM fields in the air surrounding them, which in the eddy current model is the induction effect. So the diffusion part is solved by the FEM and the induction by the BEM. In order to compute this term, we introduce an intermediate "surface current" $\vec{k}$. This surface current, which is not a physical field, is chosen such that it produces the same vector potential $\vec{A}$, and thus magnetic field $\vec{B}$ in the air $\Omega_{e}$ surrounding the conductors, as the physical volume current $\vec{j}$. This means that for any point in the air, the knowledge of this surface current is strictly equivalent to the knowledge of the actual electromagnetic fields inside the conductors. The use of a surface vector field compared to a scalar one gives flexibility to have multiply connected conductors with non simple topologies. From the Biot-Savart equation, the vector potential generated by $\vec{k}$ reads:

$$
\vec{A}(\vec{x})=\frac{\mu_{0}}{4 \pi} \int_{\Gamma_{y}} \frac{1}{\mid \vec{x}-\vec{y}) \mid} \vec{k}(\vec{y}) d \Gamma_{y}
$$

$\forall x \in \Omega_{e}$ (and thus for $x \in \Gamma$ ). 
It then can be shown that [10]:

$$
\begin{aligned}
{[\vec{n} \times(\vec{\nabla} \times \vec{A})](\vec{x}) } & =\frac{\mu_{0}}{2} \vec{k}(\vec{x}) \\
& +\frac{\mu_{0}}{4 \pi} \int_{\Gamma_{y}} \frac{1}{\mid \vec{x}-\vec{y})\left.\right|^{3}} \vec{n} \times[(\vec{x}-\vec{y}) \times \vec{k}(\vec{y})] d \Gamma_{y}
\end{aligned}
$$

In order to have a BEM with basis functions corresponding to the ones of the FEM on the surface of the conductors, thus avoiding extra matching constraints on the boundary, we still decompose $\vec{A}$ on the $\vec{W}_{1}^{\prime} s$ and introduce a set of "twisted" 1-form surface basis functions for $\vec{k}$ [10]:

$$
\begin{aligned}
& \vec{V}^{1}=\vec{n} \times \vec{W}^{1} \\
& \vec{k}=\sum_{i=1, N_{E}} k_{i} \vec{V}_{i}^{1}
\end{aligned}
$$

where $N_{E}$ is the number of boundary edges. At first order, a twisted 1-form associated with a surface edge represents a surface current flowing across the edge, i.e. with a unit surface flux across the edge and a zero surface flux across all the other surface edges. By projecting equation (18) and $\vec{k}$ on the "twisted" 1 -form basis and equation (19) against $\vec{W}^{1}$, we get a Galerkin BEM linear system which reads:

$$
\begin{gathered}
\mathbf{P} k=\mathbf{D} a \\
\mathbf{S} a=\mathbf{Q} k=\mathbf{Q}_{s} k+\mathbf{Q}_{d} k
\end{gathered}
$$

where

$$
\begin{aligned}
\mathbf{D}(i, j) & =\int_{\Gamma_{x}} \vec{V}_{i}(\vec{x}) \cdot \vec{W}_{j}(\vec{x}) d \Gamma_{x} \\
\mathbf{P}(i, j) & =\int_{\Gamma_{x}} \int_{\Gamma_{y}} \frac{1}{|\vec{x}-\vec{y}|} \vec{V}_{i}(\vec{x}) \cdot \vec{V}_{j}(\vec{y}) d \Gamma_{x} d \Gamma_{y} \\
\mathbf{Q}_{s}(i, j) & =\int_{\Gamma_{x}} \vec{W}_{i}(x) \cdot \vec{V}_{j}(x) d \Gamma_{x} \\
\mathbf{Q}_{d}(i, j) & =\int_{\Gamma_{x}} \int_{\Gamma_{y}} \frac{1}{|\vec{x}-\vec{y}|^{3}} \vec{W}_{i}(x) \cdot\left(\vec{n}_{x} \times\left[(\vec{x}-\vec{y}) \times \vec{V}_{j}(y)\right]\right) d \Gamma_{x} d \Gamma_{y}
\end{aligned}
$$

The coupled FEM+BEM system is solved at each time step by doing Richardson iterations between the FEM (17) and (23) and the BEM (22) equations until convergence:

$$
\begin{gathered}
\mathbf{P} k_{n+1}^{t+1}=\mathbf{D} a_{n}^{t+1} \\
{\left[\mathbf{M}^{1}(\sigma)+d t \mathbf{S}^{1}\left(\frac{1}{\mu}\right)\right] a_{n+1}^{t+1}=\mathbf{M}^{1}(\sigma) a^{t}-d t \mathbf{D}^{01}(\sigma) \phi^{t+1}+d t \mathbf{Q} k_{n+1}^{t+1}}
\end{gathered}
$$




\subsection{Divergence free surface current}

As already mentioned, The surface current $\vec{k}$ is an equivalent boundary current to the physical volume current $\vec{j}$ flowing through the volume of the conductors, and needs to be divergence free [10]. However, the twisted 1-forms basis functions (20) do not satisfy this divergence free constraint, since they create some net flux entering the 2 faces that lie on each side of the edge they are associated with. We first added the divergence free as an external constraint to the BEM system (22). More recently, we introduced the so-called "loop-star" solenoidal-irrotational decomposition into the divergence free "loop" basis functions and the other "star" ones $[11,12]$. As shown in Figure 2, a loop basis function associated with a node can be seen as a linear combination with coefficients +1 or -1 of twisted 1 -forms associated with all the edges originating from the node, so that it represents a (divergence-free) surface current flowing around the node.

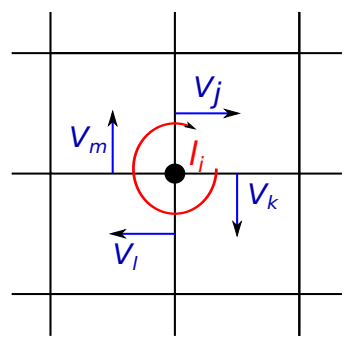

Figure 2: A loop vector basis function around node i, as the sum of the 4 twisted 1-forms $\vec{V}_{j}+\vec{V}_{k}+\vec{V}_{l}+\vec{V}_{m}$.

One can show that when using first order basis functions, the loop basis functions associated with all the nodes of the surface mesh (except one per connected part) form a complete basis of the divergence free currents for topologically simple conductors, like a sphere [11]. For non simple conductors, i.e. containing holes or "handles", a few extra non-local basis functions that we call "global currents" need to be added. For example, in the case of a prismatic conductor connected to an external circuit on each end, there is one global current representing a current flowing from one side to the other of the prism (see Figure 3). In the case of a torus, two extra global currents need to be added, one corresponding to a current flowing in the toroidal direction, and one corresponding to a current flowing in the poloidal direction.

An algorithm based on the construction of a spanning tree on the surface mesh has been developed to automatically count the number of connected parts, get their topologies by computing the "Betti numbers" [13], and in particular the number of global currents, and then set the global current basis functions as linear combinations of the 1-form basis functions. As can be seen in Figure 3, contrarily to the loop basis functions, the "global currents" are non-local basis functions 


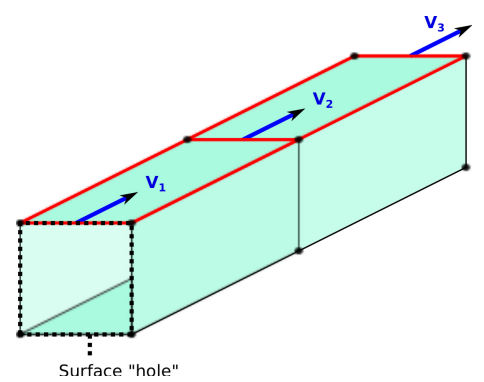

Figure 3: Global current on a prismatic conductor connected to an external circuit on each side, represented by the divergence free $\vec{V}_{1}+\vec{V}_{2}+\vec{V}_{3}$.

(hence their name), making the computation of the corresponding BEM matrices entries somewhat cumbersome. But on the bright side, the degree of freedom associated with each global current represents the total current flowing through the corresponding conductor and can be used to impose current vs time constraints as a simple dirichlet constraint in the BEM system (one dirichlet constraint per imposed current). This method allows imposing currents in geometries where more traditional methods using dirichlet conditions on the FEM system (16), (17) would require the introduction of cuts and/or multi-valued Degrees Of Freedom [14]. The above mentioned toroidal current in a torus is such an example. The use of "loop" + "global current" basis functions also gives an easy way to compute the self and mutual inductances, by solving the BEM system (22) with simple dirichlet constraints. It also allows to impose simple linear constraints between global currents like imposing that the current flowing through one conductor is equal to the one flowing through another one.

\subsection{Numerical treatment of the singular integrals}

As can be seen in (25) and (27), and as often is the case when dealing with a BEM, some integrals are singular on self and neighbor faces. Different methods to numerically treat these integrals have been proposed. For a long time, we used the simple method presented in [15] for self faces, which gives 2 sets of gaussian points as well as integration weights, hence a very simple integration scheme. We also just used the standard gauss points for neighbor faces. This method proved to be very efficient and fairly accurate for simple enough geometries with good quality meshes. Soon enough, however, customers started to use the method with elements (and hence boundary faces) with high aspect ratio, causing issues. We thus had to improve the method in order to incorporate these elongated faces, as well as handling triangular faces on top of the quadradic ones, since tetrahedra and prisms were also introduced on top of the already used hexahedra in the FEM. We are thus now using a method based on Duffy transforms $[16,17]$, which gives sets of integration points and weights for pairs of faces, either self (face against 
itself), neighbors with common edge or neighbors with common node. The scheme is slightly different depending on the type of each face (quadrilateral or triangle). We also introduced a method based on [18] in order to split faces with very large aspect ratio into better conformed ones for the computation of the corresponding singular integrals. On typical cases (around 100,000 BEM faces), the computation of the singular integrals can take up to 25 percent of the total assembly of the BEM matrices, and this process has thus been parallelized.

\subsection{Matrix storage and solve}

In order to limit the memory requirement, a domain decomposition is done on the BEM mesh, which splits the BEM matrices into blocks. On the non-diagonal blocks, a low rank approximation based on a rank revealing QR decomposition is performed, see Figure 4. For blocks corresponding to far away domains, the rank can be significantly smaller than the size of the block, thus reducing the storage of the block. We typically see reductions by factors around 20 or more between the full dense matrix and the block matrix with low rank approximations, also called Block Low Rank, or BLR [19]. As the rest of LS-DYNA, the matrix assembly is implemented in Massively Parallel Processing (MPP), where the blocks are spread between processors using a method described in [20]. The BEM system (22) is currently solved using an iterative method, Preconditioned Conjugate Gradient (PCG), where the preconditioner usually used is the diagonal block of the matrix [21]. The BLR representation of the matrix also speeds up considerably the matrix times vector operation used intensively in the PCG. A direct factorization of the BLR matrix in MPP is currently being developed at LSTC, which will allow us to use a direct solver instead of the PCG.
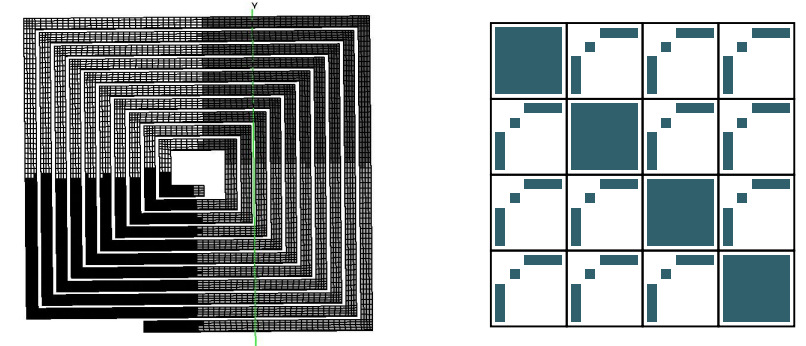

Figure 4: Domain decomposition of a BEM mesh (left) in 4 domains resulting in a four by four block matrix (right). The diagonal blocks are kept dense, and low rank approximations are performed on the others. 


\section{Features of the BEM and examples}

\subsection{Benchmark: the TEAM cases}

Along with the development of the electromagnetism module, different series of validation tests have been conducted. The TEAM (Testing Electromagnetic Analysis Methods) problems consist in a set of test-problems, with precisely defined dimensions, constitutive laws of materials, excitations and so forth, with experimental measurements on a real laboratory device [22]. The TEAM 3 problem is a classic validation test case often studied [10,23] and consists of a conducting ladder with two holes, placed below a coil carrying a sinusoidal current [22]. The coil is made of multiple turns strongly stranded together producing a uniform current while the induced current in the ladder diffuses through its thickness (the full eddy current problem is solved). The main objective of this test case is to study the behavior of the magnetic field along the symmetrical axis of the problem and between the coil and the ladder. In Figure 5, it can be observed that the biggest part of the current flows around the hole directly located under the coil. Good agreement between experimental and numerical results can also be observed. More benchmarks on various TEAM problems are presented in [24].
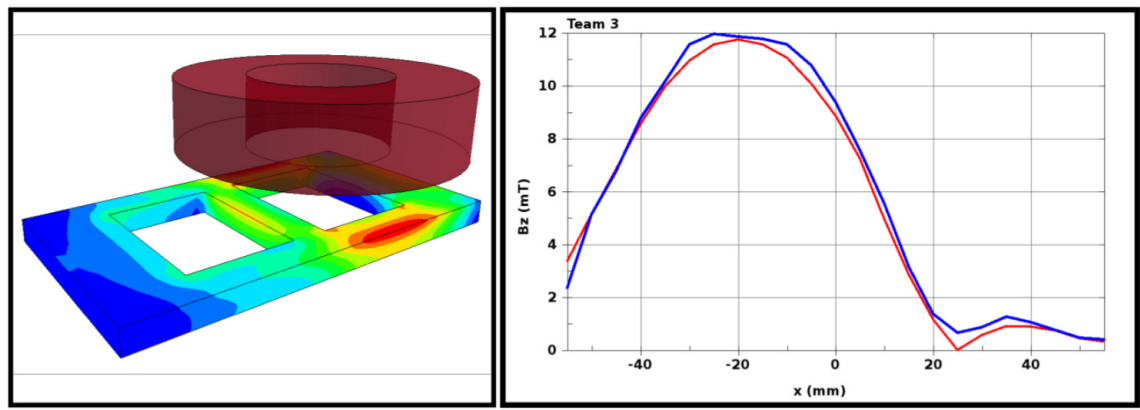

Figure 5: Current density fringes and Bz magnitude variation along the center line. Comparison between LS-DYNA (in red) and reference experimental (in blue) results.

\subsection{Small gaps and conductor motion}

Electromagnetic Metal Forming (EMF) [25], bending or welding [26] are some of the most common applications of the EM solver. EMF is a high velocity forming process where the force deforming the workpiece is a magnetic one, generated by an electrical current induced in the workpiece by a coil. Much work has shown that the formability can be significantly increased, wrinkling can be mitigated and springback can be reduced [27]. Forming of aluminum has been the main focus 
of EMF, due to its potential as a means of reducing automobile weight [28]. A typical forming setup involves one or several coils, a workpiece and a die. Strong deformations happen over a very brief period of time which the electromagnetism solver needs to be able to handle. The coil shapes are also often very complex, with several turns and with sometimes only small gaps between them, further adding to the difficulty. The case presented here features a spiral type coil and a $1 \mathrm{~mm}$ thick aluminum sheet forming on a conical die. The experiment was performed at the Department of Mechanical Engineering, University of Waterloo, Ontario, Canada [28]. Figure 6 shows the evolution of the shape of the plate.

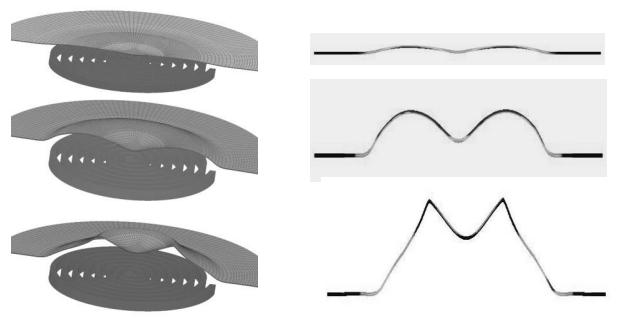

Figure 6: Magnetic Metal Forming simulation results: 3D shape of the sheet (left, only half of the sheet is represented), and current density in a cross section of the sheet at various stages during the forming process. The scale in the z-direction has been increased in the cross sections for better visibility.
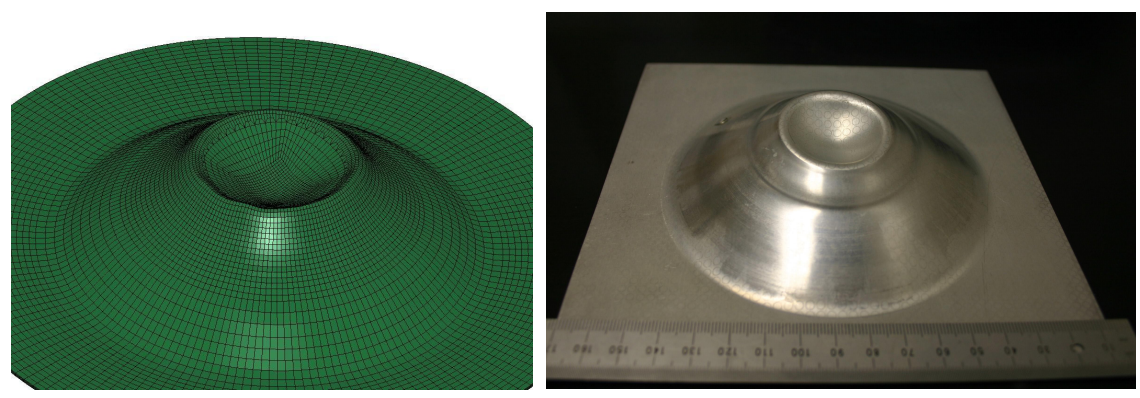

Figure 7: Magnetic Metal Forming: numerical (left) and experimental (right) final shape of the sheet.

Figure 7 shows a comparison between the numerical and experimental final shape of the sheet, which shows a very good agreement. More details on the experimental/simulation comparisons can be found in [2], and more quantitative 
benchmarks are in [29] and [30]. More recently, the eddy current model was extended to an induced heating solver. It is primarily used for industrial welding applications where the need to have spatially and temporally controlled joule heating makes the use of a simulation software very important, in order to design the heating coils, and generators [31]. The study of the over-heating and possible deterioration of the coil as well as ways to cool it down also represent an important use of the code. For slower varying fields, where the inductive effects can be neglected, a resistive heating solver was also introduced.

\subsection{Contact: local remeshing of the BEM mesh}

Since the conductors are allowed to move and deform, some contact may occur. Also, some applications like rail guns [32] need a sliding contact capability. In the FEM, the contact is handled using "mortar" like constraints, i.e. by imposing appropriate constraints on the Degrees Of Freedom (DOFs) between the 2 sides of the contact [33]. We first tried the same kind of technique in the BEM, by replacing the divergent matrix entries in the contact area by constraints between the corresponding BEM DOFs. The different constraints we tried failed to give accurate results or were not robust enough, so we finally decided to opt for a local remeshing of the BEM mesh at each time step. More specifically, in the areas where contact is detected (basically when the distance between 2 BEM faces becomes small enough), the BEM faces in contact are removed from the BEM mesh and a patented algorithm [34] allows to "stitch" the two open BEM surfaces together by building a "skirt" made of triangular faces, in order to achieve a continuous closed BEM mesh (see Figure 8). The BEM system can then be

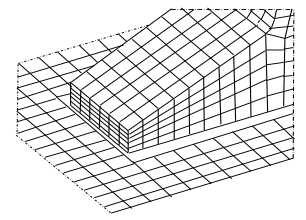

a

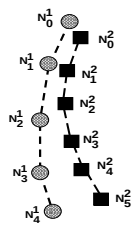

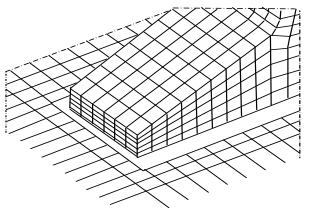

b

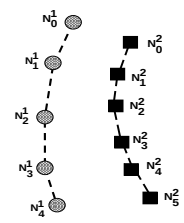

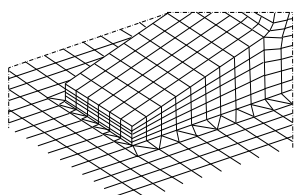

C

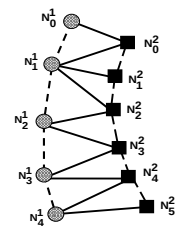

Figure 8: Remeshing of the BEM mesh around a contact area: a: The faces in contact on each side are removed. $b$ : The faces next to the contact are shrunk to ensure a sufficient gap. c: Creation of the contact "skirt" by adding triangle faces between the 2 sides of the contact. 
solved on this regular BEM mesh and appropriate constraints are added to connect the BEM DOFs to the FEM ones in the skirt areas. Figure 9 presents a rail gun application with a sliding contact. In a rail gun, the electromagnetic forces created by an electrical current are used to accelerate a projectile sliding between two conductor rails. Since the scheme depends on the current flowing between the rails and the projectile, it is a very good test for electromagnetism contact capabilities. More results on rail gun simulations are shown in [35].

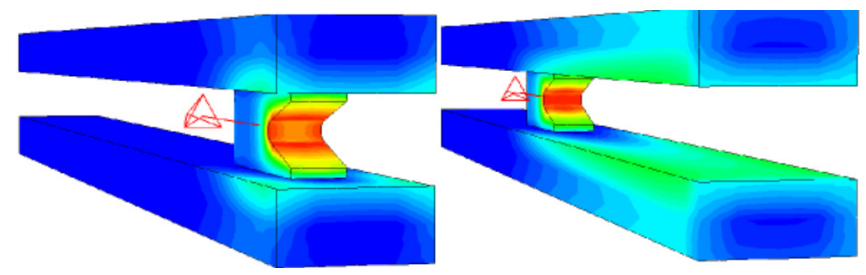

Figure 9: Railgun model showing the motion of the projectile between the 2 rails, with iso-contours of the B field.

\section{Conclusion}

We presented a BEM that, coupled with a FEM, allows to efficiently solve the Maxwell equation in the eddy current approximation, for a set of 3D multiply connected conductors. A 2D axisymmetric version also is available where, on the BEM side, the main change is in the kernel. Since it is used in a commercial code, this method has been extensively tested on all sorts of cases with different geometries and different type of meshes, and has proven very robust. The EM fields are coupled with the mechanics and the thermal part of the code, thus allowing changes in the conductor geometries and electrical conductivities, which, on the developer side, allows testing the method on different geometries within the same run. However, even with the MPP setting of the method, the BEM part of system still takes more than half of the total computational time of the EM, so a BLR factorization is being developed in order to reduce the solve time of the BEM system. The plans for the near future are the extension of the method to magnetic as well as to piezzo-electric materials, both of which should have some effect on the BEM. Longer term projects may also include the investigation of other BEM based on multipole methods.

\section{References}

[1] Hallquist, J.O., LS-DYNA Theory Manual. ISBN 0-9778540-0-0, LSTC: Livermore, CA, USA, 2006. 
[2] L'Eplattenier, P., Cook, G., Ashcraft, C., Burger, M., Imbert, J. \& Worswick, M., Introduction of an electromagnetism module in LS-DYNA for coupled mechanical-thermal-electromagnetic simulations. Steel Research Int, 80 No. 5, pp. 351-358, 2009.

[3] Biro, O. \& Preis, K., On the use of the magnetic vector potential in the finite element analysis of three-dimentional eddy currents. IEEE Transaction on Magnetics, 25 No. 4, pp. 3145-3159, 1989.

[4] Nedelec, J., A new family of mixed finite elements in R3. Num Math, 50, pp. 57-81, 1986.

[5] Castillo, P., Rieben, R. \& White, D., FEMSTER: An object oriented class library of discrete differential forms. Proceedings of the 2003 IEEE International Antennas and Propagation Symposium, 2, pp. 181-184, 2003.

[6] Rieben, R., A Novel High Order Time Domain Vector Finite Element Method for the Simulation of Electromagnetic Devices. Ph.D. thesis, Universiy of California Davis, 2004.

[7] Tonti, E., On the Geometrical Structure of Electromagnetism. Pitagora Editrice Bologna, 1999.

[8] Tonti, E., The reason for analogies between physical theories. Appl Math Modelling, 1, pp. 37-50, 1976.

[9] Rieben, R. \& White, D., Verification of high-order mixed finite element solution of transient magnetic diffusion problems. IEEE Transaction on Magnetics, 42 No. 1, pp. 25-39, 2006.

[10] Ren, Z. \& Razek, A., New technique for solving three-dimentional multiply connected eddy-current problems. IEE Proceedings, 137 No. 3, 1990.

[11] Vecchi, G., Loop-star decomposition of basis functions in the discretization of the EFIE. IEEE Transactions on Antennas and Propagation, 47 No. 2, 1999.

[12] Volakis, J. \& Davidson, D., Iterative-solver convergence for loop-star and loop-tree decompositions in method-of-moments solutions of the electricfield integral equation. IEEE Transactions on Antennas and Propagation, 46 No. 3, pp. 80-85, 2004.

[13] Bossavit, A., Computational Electromagnetism. Academic Press: Boston, 1998.

[14] Henrotte, F. \& Hameyer, K., An algorithm to construct the discrete cohomology basis functions required for magnetic scalar potential formulations without cuts. IEEE Transactions on Magnetics, 39 No. 3, pp. 1167-1170, 2003.

[15] Wang, W. \& Atalla, N., A numerical algorithm for double surface integrals over quadrilaterals with a $1 / \mathrm{r}$ singularity. Communications in Numerical Methods in Engineering, 13, pp. 885-890, 1997.

[16] Frangi, A., Novati, G., Springhetti, R. \& Rovizzi, M., 3D fracture analysis by the symmetric galerkin BEM. Computational Mechanics, 28, pp. 220-232, 2002. 
[17] Sauter, S., Cubature techniques for 3-D Galerkin BEM, Boundary Elements: Implementation and Analysis of Advanced Algorithms. W. Hackbusch, G. Wittum eds, pp. 29-44, 1996.

[18] Schwab, C., Variable order quadrature of singular and nearly singular integrals. Computing, 53, pp. 173-194, 1994.

[19] Amestoy, P., Ashcraft, C., Boiteau, O., Buttari, A., L'Excellent, J.Y. \& Weisbecker, C., Improving multifrontal methods by means of block low rank representations. SIAM, to be published.

[20] L'Eplattenier, P., Ashcraft, C. \& Ulacia, I., An MPP version of the electromagnetism module in LS-DYNA for 3D coupled mechanical-thermalelectromagnetic simulations. 11th International LS-DYNA Users conference, 2010.

[21] Chen, K., On a class of preconditioning methods for dense linear systems from boundary elements. SIAM J Sci Comput, 20 No. 2, pp. 684-698, 1998.

[22] Turner, L., Proceedings of the Vancouver TEAM workshop at the University of British Columbia. Argonne National Labs: Illinois, 1988.

[23] Zheng, D., Three dimensional eddy current analysis by the boundary element method. IEEE Transactions on Magnetics, 33, pp. 1354-1357, 1997.

[24] Çaldichoury, I. \& L'Eplattenier, P., Validation process of the electromagnetism (EM) solver in LS-DYNA v980: The TEAM problems. 12th International LS-DYNA Users conference, 2012.

[25] Boyd, J.I., Hybrid Electromagnetic Forming of Aluminum Alloy Sheet. Ph.D. thesis, Universiy of Waterloo, 2010.

[26] Berlin, A., Nguyen, T., Worswick, M. \& Zhou, Y., Metallurgical analysis of magnetic pulse welds of AZ31 magnesium alloy. Science and Technology of Welding and Joining, 16 No. 8, pp. 728-734, 2011.

[27] Balanethiram, V. \& Daehn, G., Hyperplasticity: increased forming limits at high workpiece velocity. Scripta Metallurgica Et Materialia, 30, 1994.

[28] Boyd, J.I., Increased Formability and the effects of the Tool/Sheet Interaction in Electromagnetic Forming of Aluminum Alloy sheet. Master's thesis, Universiy of Waterloo, 2006.

[29] Ulacia, I., Imbert, J., L’Eplattenier, P., Hurtado, I. \& Worswick, M., Numerical simulation of electromagnetic forming process using a combination of BEM and FEM. Numisheet 2008, Interlaken, Switzerland, 2008.

[30] Ulacia, I., Temperature dependent high strain rate deformation mechanisms in AZ31B Mg alloy and coupled numerical modeling of electromagnetic forming process. Ph.D. thesis, Universiy of Mondragon, Spain, 2009.

[31] Duhovic, M., Moser, L., Mitschang, P., Maier, M., Çaldichoury, I. \& L'Eplattenier, P., Simulationg the joining of composite materials by electromagnetic induction. 12th International LS-DYNA users conference, 2012.

[32] Krause, P., Wasynczuk, O. \& Sudhoff, S., Analysis of Electric Machinery. IEE Press, 1995. 
[33] Rapetti, F., Bouillault, F., Santandrea, L., Buffa, A., Maday, Y. \& Razek, A., Calculation of eddy currents with edge elements on non-matching grids in moving structures. IEEE Transactions on Magnetics, 38 No. 4, pp. 1351$1355,2000$.

[34] L'Eplattenier, P., FEM-BEM coupling methods and systems for sliding contact interface, US patent 8,489,372 B2, July 16, 2013.

[35] Çaldichoury, I. \& L'Eplattenier, P., Simulation of a railgun: A contribution to the validation of the electromagnetism module in LS-DYNA v980. 12th International LS-DYNA Users conference, 2012. 